\title{
TENDENCIAS POSTMODERNISTAS \\ EN EL PENSAMIENTO ORGANIZACIONAL
}

\section{Jenny K. Rodríguez Ortiz}

"En realidad existen casos en los que se tiene delante el sentido de lo que se quiere decir mucho más claramente de lo que se puede expresar con palabras."

$$
\text { Ludwig Wittgenstein }
$$

“ 'Oh fangosa grandeza! ¡Oh sublime ignominia!”

Baudelaire

\section{RESUMEN}

Se analiza el discurso de la postmedernidad a partir de tres ejes fundamentales y cómo se ven reflejadas estas ideas de los escenarios organizacionales, sobre todo en la naturaleza de la realidad y de la producción del conocimiento. Luego se estudian cuatro enfoques de la teoría organizacional postmodernista: la teorización feminista post-estructuralista, los análisis post-coloniales, la teoría actor-red y los enfoques narrativos del conocimiento.

\section{PALABRAS CLAVES}

Postmodernismo, pensamiento organizacional, teorización feminista, análisis postcoloniales, teoría actor-red, enfoques narrativos.

* PhD (c) in Development Administration and Management. Institute for Development Policy and Management (IDPM). The University of Manchester: Reino Unido. La autora pertenece a la Facultad de Administración y Economia (FAE) de la Universidad de Santiago de Chile. 
La evolución del pensamiento organizacional ha ido de la mano con los cambios sociales, económicos, políticos y tecnológicos experimentados por la humanidad. En alguna medida, el conocimiento organizacional se reconstruye en el marco del amplio espectro que supone pensar el quehacer organizacional. ${ }^{\prime}$ En este sentido, la tarea de la teorización organizacional ha sido la de articular un discurso coherente que explique, en el marco de las contingencias temporales, el pensamiento y acción en materia de espacios y dinámicas organizacionales.

En la actualidad, se argumenta que somos parte del autodenominado discurso de la postmodernidad, que para unos representa ideas y valores compartidos por grupos sociales, y para otros representa una concepción errónea de la realidad social (Lyon, 1997; Dellamora, 1995; Jameson, 1991). Este discurso encuentra sustento en tres ejes fundamentales que guían el ser y el hacer: la reivindicación de lo plural y lo particular, el rechazo de la filosofía del sujeto y la crítica de la razón a partir de un escenario anarquista de ésta (López Gil, 1993).

La reivindicación de lo plural y lo particular subraya la importancia del contextualismo y la desconfianza frente a sistematizaciones muy fuertes y generalizaciones excesivas. El rechazo a la filosofía del sujeto llama a una clara oposición al dualismo cartesiano (mente/cuerpo) a través de la inclusión de otras categorías como acción, lenguaje y espíritu. Y la crítica de la razón se sustenta en el abandono del pensamiento metódico, la responsabilidad teórica y el igualitarismo científico. En resumen, y tal como plantea Glucksmann (1997), se identifica una doble atomización del orden de las ideas y de la conexión de las cosas.

1 Esto resulta especialmente evidente si se considera la gran cantidad de producción y teoría sobre la organización, así como la amplitud en su diversidad temática. 
A propósito de este discurso postmodernista, algunos autores (Hopenhayn, 1992, 2000; Touraine, 1990;) se refieren a la muerte cultural de la sociedad, la brecha entre la integración simbólica y la material, la orfandad existencial, la presión hacia el pluralismo de las formas de vida y la dicotomía entre la subyugación/opresión/represión y la liberación que tanto se replantea y en la cual se resume el aire postmodernista.

Estas ideas se ven reflejadas en los escenarios organizacionales de diversas formas; por una parte, en nuevas formas de interpretación y reinterpretación de estos escenarios, y por otra, en nuevas formas de ver y comprender la organización del trabajo y, a partir de ello, re-conceptualizar los supuestos sobre la naturaleza de la realidad y de la producción del conocimiento.

En general, subyacen ideas encontradas en un punto, lo cual sugiere el rompimiento con las lógicas de pensamiento, el surgimiento de nuevas políticas culturales que abren espacios para voces no tradicionales y actores diversos, y el conflicto que generan ambas cosas frente al tradicionalismo societal (Friedman, 2001). En el caso organizacional, el rompimiento iría de la mano con la forma en que se conceptualizan, estudian y analizan las problemáticas al interior de las organizaciones.

A partir de esto se ha estimulado una reflexión más filosófica sobre la cognición y la conducta en las organizaciones, orientándose a un análisis epistemológico de las bases de la teorización organizacional. Hancock \& Tyler sostienen que "el postmodernismo ha ayudado a mover el estudio de las organizaciones más allá de las rígidas demarcaciones ontológicas entre el objeto y el sujeto de investigación y ha generado interés por la investigación de aspectos de la vida organizacional previamente juzgados, a lo más, como periféricos y, a lo menos, como completamente inapropiados para una consideración científica social seria" (2001:63). 
Es en este marco donde se han desarrollado los cuestionamientos epistemológicos contemporáneos relativos a la producción de conocimiento. En este sentido, un espacio discursivo importante corresponde a los denominados estudios postmodernistas de la organización, que se caracterizan por una preocupación primordial por el lenguaje y la representación, y una reconsideración de la subjetividad y el poder (Calas \& Smircich, 1999), así como una problematización del sujeto y del autor. Esto es de particular importancia en la teorización organizacional; ha llevado al cuestionamiento de la construcción del conocimiento, porque las formas en que este conocimiento es utilizado para categorizar los fenómenos que operan en los entornos organizacionales son muchas veces tomadas como pilar axiomático para gestionar estos entornos. ${ }^{2}$

Al hablar de la reconsideración de la subjetividad y el poder, nos referimos a la tendencia que refiere Spender (1998) de una comprensión reflexiva de la teorización en estudios organizacionales como un proceso político y no sólo como un proceso neutral de búsqueda de la verdad. La idea que rodea a estas nuevas tendencias es una reflexión hacia la constitución de "teoría" y la consideración de los aspectos institucionales, sociales y políticos de tal constitución. Su finalidad, sostiene Van Maanen (1988), se resume en la reflexión sobre la producción de teoría como un género literario y como una actividad cultural e institucional. Estas tendencias teóricas enfatizan la relación entre "poder" y "conocimiento" en la configuración de la teoría, abriendo espacio a la consideración de los problemas de exclusión, representación y forma, en una suerte de poética de la construcción del conocimiento (Foucault, 1984).

2 Crook, Pakulski \& Waters (1992) refieren la fragmentación que yace en el centro del proceso de post-modernización y que se manifiesta en todo el espectro organizacional. 
Podría resumirse que el problema del conocimiento en la postmodernidad tiene su raíz principal en el cuestionamiento de la visión modernista con relación a la postura universalista respecto de lo verdadero, lo bueno y lo bello. ${ }^{3}$ A partir de la defensa de los planteamientos postmodernistas sobre la importancia de la localización en el tiempo y el espacio, y la capacidad de adaptación y desaparición si fuese necesario, el paradigma dominante de funcionalismo - positivismo es cuestionado por perspectivas interpretativas y críticas que representan otros juegos de lenguaje.

Según Lyotard (1984), el postmodernismo como forma de conocimiento reconoce que las historias sobre verdad, progreso y eventual emancipación no son sino narrativas históricas. De ahí la necesidad de búsqueda de nuevas formas de representación del conocimiento y la verdad; formas que den cuenta de la fragilidad de las bases ontológicas, epistemológicas y éticas del tiempo presente.

Una primera aproximación al fenómeno de la postmodernidad en los entornos organizacionales se referiría a lo sostenido por Heydebrand sobre un "nuevo modo de administración" (1989:339). Esta postura es compleja ya que, en tanto podría pensarse que la postmodernidad organizacional está dada por el determinismo tecnológico e institucional, los debates de mayor fuerza (Clegg, 1993) sugieren que tanto los desarrollos globales culturales y tecnológicos así como las luchas de significado y poder ejercen una influencia determinante.

En un interesante escrito, Cooper \& Burrell (1988) plantean que, mientras los enfoques modernistas para estudio de la vida organizacional presentan las organizaciones como fenómenos inmutables, como extensiones de la racionalidad humana, los

3 Para Lyotard (1984), el postmodernismo es, principalmente, una reacción paralogística al modernismo monológico. 
enfoques postmodernistas abordan las organizaciones como expresiones contingentes y temporales de un impulso modernista sistémico a ordenar las relaciones sociales de acuerdo con el modelo de racionalidad funcional. Esto es, se entienden las organizaciones como "reacciones defensivas contra las fuerzas del caos” (p. 91)

Los enfoques contemporáneos en la teorización organizacional se concentran en el rechazo de las metanarrativas, y de la continua pregunta de cómo escribir conocimiento legítimo en la postmodernidad. Taylor (1984) sostiene que este rechazo se debe a que las metanarrativas plantean una historia definitiva; esto es, con un inicio y un fin. Esto, en un mundo postmoderno, es imposible y no tiene ningún sentido.

En este sentido, la teorización es tanto una manifestación de, como una contribución a lo que Habermas (1975) ha llamado la crisis de legitimación en la cultura occidental y que se contrapone a lo que Lyotard (1984) identifica como las grannarrativas de legitimación o las metanarrativas de la civilización occidental. ${ }^{4}$

Centrando la atención en la textualidad de las teorías organizacionales, el postmodernismo ha abierto un espacio para una forma distinta de crítica; una crítica que se sustenta, primero, en el supuesto de que la teoría es una forma representacional que define "para qué" y "para quiénes" se habla desde la escolaridad (Fondas, 1997; Putnam, 1996; Van Maanen, 1988)

4 En su escrito The Postmodern Condition: A Report on Knowledge, Lyotard (1984) popularizó el término postmodernismo al interior de la filosofia y las ciencias sociales, y expuso su visión de que el modernismo, como un fenómeno intelectual y cultural, era un conjunto de lo que denominó metanarrativas (xxix), las cuales describen cualquier forma de narrativa utilizada para legitimar declaraciones o alegatos respecto del conocimiento o la acción. Son estas metanarrativas las que han amparado y legitimado históricamente la postura del hombre occidental de auto-imponerse la misión de transformar el planeta según su propia imagen. 
y segundo, en una respuesta a la multiplicidad paradigmática que subyace a la teoría organizacional (Chia, 1997).

Esta respuesta, sin embargo, no es un cuestionamiento al hecho de que exista esa multiplicidad, sino que más bien se relaciona con lo que subyace al hecho de que lo que sustenta esos diversos paradigmas sea o no conocimiento verdadero, ya que cada paradigma (o metateoría) es un postulado fundacional sobre la posibilidad de dicho conocimiento verdadero. ${ }^{5}$

Se ha sostenido (Hancock \& Tyler, 2001; Calas \& Smircich, 1999; Burrell, 1994, 1996, 1997) que el análisis organizacional postmodernista subyace en la teorización que aborda cómo la reflexividad (la ontología crítica sobre nosotros mismos), la diferencia y el cambio (la transgresión de las restricciones internas de la subjetividad y la subjetivación) pueden reconstituir las formas en que se reproduce el conocimiento organizacional. Alvesson \& Willmott (1996) sugieren que, dado que la reflexión postmodernista ha abierto un espacio a la Teoría Crítica, se puede hacer uso de ésta para llegar a una praxis emancipatoria en la administración. Esto es, a partir de una crítica importante de la práctica gerencial como una reflexión de un cierto tipo de teoría de la administración, se pueden indicar caminos alternativos a seguir para superar las disfunciones de la definición dominante de administración.

Cuatro enfoques fundamentales son reconocidos como pilares de la teorización organizacional postmodernista (Calas \& Smircich, 1999); la teorización feminista post-estructuralista,

5 Es precisamente esto lo que ha llevado a algunos autores (Parker, 2000; Alvesson, 1995; Thompson, 1993) a cuestionar la base empírica de la propuesta de teorización organizacional postmodernista, acusándola de ser meramente una técnica semántica, un vano esfuerzo académico para hacer surgir nuevos dominios de cuestionamiento que luzcan noveles y dignos de atención. Esto porque se considera que la propuesta es poco clara conceptualmente en sus esfuerzos de definición e identificación de las organizaciones postmodernizadas. 
los análisis post-coloniales, la teoría actor-red y los enfoques narrativos del conocimiento. Estos análisis podrían ser identificados como la resistencia que refiere Foucault (1984) en sus trabajos sobre la crítica genealógica, ya que un punto común en estos enfoques es el interés en desestabilizar las estructuras de reconocimiento que constituyen nuestras subjetividades y hacer uso de la auto-representación creativa para resistir las condiciones organizacionales que restringen la posibilidad de autonomía individual.

\section{Teorización feminista post-estructuralista}

El postmodernismo es un espacio de gran significación para la teorización feminista, ya que en tanto busca desarrollar una comprensión libre de verdades y de teorías, hace más factible la permanencia en el espacio de la teorización flexible, que tantos problemas ha traído a la teorización en ciencias sociales, y en especial a la teorización feminista.

Rosenau (1992:86) sostiene que "el campo de los estudios de mujeres confronta el dilema planteado a cualquier disciplina de la ciencia social por parte de las versiones postmodernas de verdad y teoría”. En este sentido, la lucha feminista contra la teorización, a la que acusa de patriarcal, encuentra asidero en el cuestionamiento postmodernista a las comunidades de conocimiento que plantean y presentan verdades con cierto grado de soberbio absolutismo.

La emergencia del feminismo post-estructuralista (también llamado feminismo postmodernista y post-feminismo) surge como resultado de la evolución desde las perspectivas anteriores que, inicialmente, se sustentaban en posturas pro-femeninas, pero cuya teorización en la actualidad incluye lo masculino, las masculinidades, así como también cruces transversales 
con variables como la diversidad, raza, religión, clase, entre otras. Lo anterior habla claramente de la consideración y reconstrucción de los grupos considerados como marginados o silenciados por los poderes hegemónicos y, de algún modo, denuncia la androcentricidad de la ciencia que refiere Duran (1998). ${ }^{6}$

Es evidente que, a partir de este importante paso, el foco epistemológico de la teorización feminista se vio sometido a escrutinio interno, lo que conllevó el desarrollo de nuevas formas de reflexión y análisis del fenómeno. Es así como se comienza a hablar de teorización feminista post-estructuralista la que, aun cuando inició su despegue en los años '80, ganó popularidad en los años '90, cuando el post-estructuralismo abrió el espacio para considerar el género teóricamente, independiente de cuerpos sexuados particulares, generando un encuentro entre la teoría feminista y los postulados de deconstrucción y genealogías del postmodernismo, por cuanto estas últimas explicitan la devaluación de lo femenino en las teorías "universales" y las prácticas discursivas.

Sin embargo, no puede comprenderse la importancia de la teorización feminista post-estructuralista sin antes hablar de la evolución histórica de la teorización feminista. Chafetz define una teoría como feminista "si puede ser usada (sin importar por quién) para cuestionar, contraatacar o cambiar un estatus quo que devalúa u opera en desventaja de las mujeres" (1988:5). Históricamente, el feminismo ha sido asociado con la lucha por la independencia política, social y económica de las mujeres; sin embargo, en el marco de la evolución de la

6 Para este fin particular, establece un sinnúmero de cadenas interdisciplinarias que incluyen la lingüística, la antropología estructural y la historia, el psicoanálisis y la deconstrucción o filosofía del límite a fin de re-conceptualizar las verdades que excluyen a muchos "otros" a partir de la relación de esas verdades con esos "otros". 
teorización feminista, la palabra mujer ha designado muchas cosas: sexo, género, clase, sexualidad, edad, entre otras.

Estas designaciones responden a las posiciones al interior del feminismo, las que pueden ser identificadas y clasificadas en diversas formas. Una de ellas es de acuerdo a la postura política. ${ }^{7}$ Encontramos, por ejemplo, el feminismo liberal (que aboga por la igualdad de género), el feminismo radical (que rechaza la sociedad dominada por lo masculino como un todo y aboga porque las mujeres transformen radicalmente el orden social existente y desarrollen sus propias instituciones sociales) y el feminismo socialista (inspirado en la teoría marxista y que aboga por el desarrollo de estudios críticos de la sociedad con la idea de contribuir a un cambio radical donde nuevas relaciones de género sean incluidas como elementos centrales). En general, estos enfoques se refieren a la resistencia contra la tiranía del discurso monolítico (Donovan, 1996), foco central subyacente a la teorización feminista.

Sin embargo, bajo estos paradigmas feministas tradicionales, los temas de raza y diferencia se veían opacados por la categoría de sexo. ${ }^{8}$ Es a raíz de la transición desde la problemática de mujeres a la problemática de género que el espectro analítico se amplía significativamente. Alvesson \& Due Billing (1997) sostienen que los estudios de género han evolucionado alrededor de tres puntos fundamentales:

1 ro. Que el género es central y relevante para entender las relaciones, instituciones y procesos sociales.

7 Las teorías feministas se han caracterizado por ser siempre politicas, independientemente de las filosofias que profesen y mayormente tratan de cómo y por qué ocurre la exclusión u opresión de la mujer y cómo proveer remedios para esta situación.

8 Un supuesto de la modernidad respecto del cuerpo y la sexualidad es que el primero expresa una verdad fundamental sobre el segundo. 
2do. Que las relaciones de género constituyen un problema en tanto están caracterizadas mediante patrones de dominación/subordinación, inequidades, opresiones y oposiciones.

3ro. Que las relaciones de género son vistas como construcciones sociales.

Al hablar de género ya no nos referimos exclusivamente al problema de las mujeres, sino al problema de los géneros. $Y$ es precisamente en este contexto donde encuentran espacio nuevas formas de teorización feminista.

El post-estructuralismo, por su parte, se refiere al desarollo de una teoría crítica que emergió después del estructuralismo ${ }^{9}$ y que, en algún sentido, surgió como una rebelión frente a éste. En algunos casos, el término es utilizado en forma intercambiable con el término postmodernismo. ${ }^{10} \mathrm{El}$ postestructuralismo representa, al mismo tiempo, un desarrollo y una deconstrucción del estructuralismo y puede ser entendido como un enfoque filosófico que aborda la relación entre el sujeto humano y las fuerzas estructurales, tales como la cultura, el lenguaje y la tradición (Hancock \& Tyler, 2001; Hawthorn, 1992).

Como movimiento, provee una base filosófica para la crítica postmodernista a los postulados absolutos sobre el conocimiento, que sirven de sustento a la cosmovisión modernista. En forma alternativa, el post-estructuralismo considera que el

9 El estructuralismo surge del funcionalismo estructural y se centra en el establecimiento de interconexiones entre partes que, necesariamente, son a su vez parte de un todo complejo. Andermahr, Lovell \& Wolkowitz (2000:263) plantean que el concepto es "una metáfora arquitectónica que ha permeado las ciencias $y$ las humanidades " y que se centra en mantener un estatus científico riguroso.

10 Aun cuando este último se refiera más certeramente al movimiento en las artes que sucedió al modernismo. 
mundo, incluyendo la experiencia subjetiva al interior de éste, es el resultado del lenguaje. ${ }^{11}$

En el marco de sus planteamientos, el post-estructuralismo abre un espacio importante para estas nuevas formas de teorización feminista, ya que propicia no sólo el escrutinio interno de sus postulados, sino también el desarrollo de una teorización con mayor fuerza argumentativa frente a la teorización que tradicionalmente la excluye y subordina.

Como sostiene Smith (1995), mucho ha sucedido desde que a mediados de los años ' 80 la historiadora Joan Scott propuso que la categoría de mujer fuera reemplazada por la de género. En la actualidad, ya se habla de una perspectiva de género, lo que ha resultado positivo desde el punto de vista analítico, ya que ha ubicado el esfuerzo de la teorización feminista en un nivel más alto de sensibilidad respecto de las contradicciones y ambigüedades relativas a las construcciones y reconstrucciones sociales de las relaciones de género. Igualmente, renuncia a tratar los temas de género como exclusivos de la dicotomía sexual hombre/mujer, abriendo espacios a todas las construcciones genéricas posibles.

Sin embargo, cabe destacar que la legitimidad de este espacio sigue siendo controvertida, ya que muchos de los principales arquitectos (masculinos) del postmodernismo y del post-estructuralismo con frecuencia han rechazado el feminismo (Andermahr, Lovell \& Wolkowitz, 2000), lo que ha

11 Para los post-estructuralistas, no se pueden descubrir verdades o postulados esenciales que gobiernen el orden social y sus sistemas institucionales. Sostienen que, dado que la tarea de asignar significado a los fenómenos es intrínsecamente dependiente del lenguaje, y dado que actos como nombrar, describir, interpretar y comprender son procesos completamente subjetivos, la tarea de asignar significado no es más que un proceso arbitrario (subjetivo) de selección. O sea, depende sólo de que la gente comparta significados similares. 
llevado a que el feminismo postmodernista sea considerado como una apropiación. ${ }^{2}$

Por otra parte, dada la invisibilidad sistemática que sufre su teorización, las feministas necesariamente luchan por la superioridad de su propio punto de vista, lo que también lleva a complicar la presencia de esta teorización en el marco de los postulados postmodernistas. El postmodernismo rechaza toda posibilidad de teoría (Weedon, 1987) y plantea que todas las perspectivas son arbitrarias, incluyendo la feminista, abogando por la aceptación igualitaria de todas las versiones de verdad. Estas ideas son complicadas para el feminismo porque, tal como plantea Harding (1990), el feminismo plantea que las mujeres necesitan una epistemología propia donde el conocimiento sea posible y sus interpretaciones de éste válidas.

\section{Análisis post-coloniales}

El término post-colonial es conflictivo, ya que no hay acuerdo sobre la validez de lo que se deriva de la conceptualización y análisis desde/hacia la post-colonialidad. ${ }^{13}$ Según Roy (2001), el concepto surgió en medio de crecientes sentimientos de impotencia y pesimismo por parte de los intelectuales de los países del denominado tercer mundo. La idea de que

12 El término se aplica a las "invasiones" a la teoría para obtener botines conceptuales útiles. "Las estrategias de apropiación postmodernistas han legitimado la práctica de invadir teorias que. con frecuencia, son completamente inocentes de involucramiento en los asuntos feministas, transformándolas con el propósito de desarrollar una teoria feminista". (Andermahr, Lovell \& Wolkowitz, 2000:15).

13 Hay fuertes debates sobre al uso de los términos anti-colonial, contra-colonial y postcolonial, así como de la pertinencia de hablar de éstos como discursos que son independientes unos de otros. Roy (2001) refiere la condenación de algunos críticos respecto de la adecuación del término post-colonial en tanto nombra un globo terráqueo no del todo descolonizado. 
estas tendencias emergieran de los académicos del denominado tercer mundo influye significativamente en la forma inicial que tomaron estos análisis, que se centraba en el desarrollo de un movimiento anti-colonialista. ${ }^{14}$

Una parte importante del movimiento se origina en el cuestionamiento de los postulados del conocimiento moderno occidental, en virtud de que silencia las voces marginales. Cabe destacar que el post-estructuralismo abre el espacio para estos cuestionamientos, por cuanto en sí mismo es una crítica a la epistemología de occidente a lo que conceptualiza como un sistema de exclusiones. En el "Manifiesto Inaugural" del Grupo Latinoamericano de Estudios Subalternos (Castro-Gómez, 1998), se asocia el problema principal de la post-colonialidad con lo referido por Guha con relación a la incapacidad histórica de las naciones para auto-realizarse, lo que se deriva del liderazgo inadecuado de sus élites gobernantes. ${ }^{15}$

La teorización crítica post-colonial se centra en el cuestionamiento de la evidencia histórica con el objetivo de presentar una visión más compleja de la identidad, una visión que supere la dicotomía dominador/dominado. Los académicos postcoloniales analizan las intersecciones de las teorías e instituciones occidentales como políticas de conocimiento y parten de la base de que nunca se han realizado esfuerzos suficientes para distinguir la gran heterogeneidad del denominado tercer mundo (Paolini, 2000).

En sus inicios, las propuestas post-coloniales se centraban en un énfasis en la subjetividad, una crítica a la modernidad, un debate sobre el concepto de Estado - Nación y el compromiso

14 Pareciera ser que la conciencia de la colonización teórica generó sentimientos nacionalistas en estos intelectuales, los que resultaron en fuertes consignas anticolonialistas.

15 Esto refiere en forma particular los trabajos sobre estudios subalternos desarrollados por Guha \& Spivak en 1988 (ver Castro-Gómez, 1998). 
con lo marginal. Puede argumentarse que el post-colonialismo estuvo preocupado de generalizar, identificar temas amplios y patrones recurrentes sobre la base de recuentos específicos de conducta personal, práctica política y formaciones culturales (Darby, 2000).

Posteriormente, ocurren algunos cambios sustanciales en la orientación del discurso, los cuales pueden ser resumidos en:

1 ro. Movimiento desde lo personal y lo particular hacia los análisis abstractos.

2do. Movimiento desde la resistencia y la recuperación hacia la ambivalencia y la hibridez.

3ro. Movimiento desde las experiencias y preocupaciones esencialmente tercermundistas hacia perspectivas globalizadas.

Es así como las críticas post-coloniales se extendieron también a narrativas sobre los orígenes en las teorías occidentales, abordando el problema político de la legitimidad de los argumentos actuales sobre la constitución de los grupos humanos y su segmentación (Scott, 1999), así como las identidades, las diásporas, la migración, la transnacionalidad, el localismo y la post-nacionalidad. A raíz de esto se presentan reconceptualizaciones que ponen en tela de juicio la base que define algunas categorías históricas; como por ejemplo, hablar de modernidad alternativa (Ong, 1996) más que de modernidad tardía.

Un aspecto importante es que los análisis post-coloniales centran sus propuestas en la necesidad de enfatizar el fraccionalismo en el desarrollo político e histórico de las regiones del denominado tercer mundo y el consecuente mosaico de construcciones diversas que deben considerarse a fin de teorizar sobre las formas en que se articula el conocimiento. Por ejemplo, lo global enmarca la identidad en muchos lugares del denominado tercer mundo; sin embargo, no contempla ne- 
cesariamente la forma en que los discursos de globalización operan con relación al supuesto de la homogeneización que subyace a la occidentalización.

En este sentido, las heterogeneidades son un punto central de la condición post-colonial. La teoría occidental hegemónica ha creado contextos basados en un sentido unilateral de la historicidad, lo que, a partir de la crisis de conciencia desde donde emergen los análisis post-coloniales, ha generado esa necesidad que refiere Spivak (1988) de comprender los límites históricos y conceptuales de la invención colonial de la tradición. ${ }^{16}$

El corpus teórico post-colonial hace uso de los análisis marxistas de narrativa, basándose en informaciones históricas y políticas que dan cuenta de los supuestos asumidos en relación a la colonialidad. Esto se relaciona con una crítica a la complicidad teórica entre diversas narrativas del estatus quo y el auto-examen de las supuestas formas de resistencias al interior de las estructuras de poder. ${ }^{17}$

La intencionalidad de la teorización post-colonialista retrabaja en forma narrativa las fuerzas ideológicas que operan en la creación de imaginarios de mundo e identidad de las comunidades colonizadas y persigue desenmascarar las estrategias de desfamiliarización usadas para invisibilizar las representaciones diversas de las comunidades subalternas. Esto es, la teorización de los procesos de tradición, resistencia, di-

16 Tal como refiere Spivak (1988), la gente fuera del Primer Mundo tiene una utilidad continua, ya que el sujeto etnocéntrico se establece a sí mismo selectivamente definiendo un otro.

17 Hablar de'supuestos', más que evidenciar un esfuerzo de perverso escepticismo, responde más bien a la consideración de que, tal como argumenta Darby, "el sujeto colonizado nunca fue completamente resistente a los encantos e incentivos del proyecto imperial. Inevitablemente, algunos de los valores de fuera tomaron raiz al interior de las sociedades coloniales" (2000:18) 
ferencia, hibridez, cambio y apropiación, en tanto intensos y ambiguos, son esencialmente diferenciadores de los contextos del denominado tercer mundo.

\section{Teoría actor-red}

El planteamiento central de la teoría actor-red es que existen redes heterogéneas de intereses alineados (Callon \& Latour, 1981); esto es, los individuos forman alianzas, atraen a otros actores, y usan artefactos para fortalecer tales alianzas y asegurar sus intereses, creando entonces redes de humanos y artefactos.

Se ha planteado (ver Latour citado en Stark, 2001) que la teoría actor-red surge del concepto de "rizoma" en el trabajo de Deleuze \& Guattari ${ }^{18}$ y que puede ser entendida como una semiótica de la materialidad. La perspectiva de actores en red emerge como una alternativa al episteme moderno y propone un cambio teórico en el énfasis, alejándolo de la centralidad y primacía del sujeto humano (Somerville, 1999); esto es, trata al sujeto humano simplemente como un actor en una red. Es una teoría reflexiva que se concentra en el irreduccionismo y en la racionalidad, más que en hechos y esencias.

Esta teoría fue concebida por Callon (1986), Callon \& Latour (1981), Latour (1992) y Law (1992), quienes también la han desarrollado exhaustivamente. Apareció por primera vez en los estudios sociales de ciencia y tecnología, y sus orígenes están mezclados con otras ciencias que incluyen, entre otras, semiótica / estructuralismo, fenomenología y

18 Se refiere al trabajo de Deleuze y Guattari (1988) donde abordan los conceptos de desterritorialización y nomadismo, entre otros. Estas nociones, de alguna manera, se asocian con la idea de red que plantea esta teoría, en tanto esta es una cadena dispersa y descentrada de actividades ocurrentes y mutantes. 
etnometodología. ${ }^{19}$ Esta teoría es un enfoque sociotécnico que, según Kaghan \& Bowker (2001), puede ser vista como una respuesta a la investigación racionalista / funcionalista de sistemas sociotécnicos grandes y como una extensión y explicación de los marcos estructurales pragmáticos / culturalistas desarrollados en sociología y antropología.

El constructo central de la teoría es el actor, que puede ser humano o no-humano y que es reconocido como cualquier elemento que modela el espacio a su alrededor, hace a otros elementos dependientes de él y traduce sus deseos en un lenguaje propio. Varios conceptos sustentan esta dinámica; entre otros, la problematización, el interesamiento y el enrolamiento, los cuales explican la interacción de un actor focal con otros actores desde el primer momento de relación (problematización) hasta el momento concluyente (enrolamiento). Simultáneamente con el desarrollo de la dinámica, operan otros procesos como el punto de paso obligatorio, la inscripción, la traición y la irreversibilidad.

Podría resumirse el proceso planteado por la teoría actorred, más o menos como sigue: un actor define identidades e intereses de otros actores, que son consistentes con sus propios intereses (problematización) y se establece como un punto de paso obligatorio en tanto se hace indispensable para la dinámica que genera para el resto. Luego procede a convencer a los actores a aceptar los intereses definidos por él para ellos (interesamiento) ${ }^{20}$ Este convencimiento tiene una connota-

19 Este punto es abordado por Gomart \& Hennion (en Law \& Hassard, 1999), quienes plantean la necesidad de una "teoría de red de eventos", cambiando de "quién actúa" a "lo que ocurre". Estos autores sostienen que esta teoría, más que una teoría es un método de ser fiel a los principios de la etnometodología, pues los actores saben lo que hacen y nosotros tenemos que aprender de ellos no sólo lo que hacen, sino cómo y cuándo lo hacen.

20 El punto de paso obligatorio es una situación o proceso que debe ocurrir a fin de que todos los actores satisfagan sus intereses, los que les han sido atribuidos por el actor focal. 
ción de negociación, la cual puede llevarse a cabo entre ellos o entre el actor focal y representantes que son enviados por los otros actores para que negocien en su nombre. Si el interesamiento es exitoso, ocurre el enrolamiento, que involucra una definición de roles de cada uno de los actores en la recién creada red de actores. De lo anterior se deriva la creación de artefactos técnicos que aseguren la protección de ciertos intereses comunes (inscripción). Sin embargo, el proceso puede verse afectado si los actores no cumplen con los acuerdos logrados por sus representantes (traición), lo que puede llevar a un punto donde no sea factible retomar otras alternativas previamente existentes (irreversibilidad). ${ }^{21}$

El enfoque actor-red supone que ningún actor es pasivo; sin embargo, aun cuando todos tienen cierto grado de injerencia, todos varían en la forma e intensidad en que influyen a otras entidades y en que resisten la influencia de otras entidades. Tanto micro-actores como macro-actores ${ }^{22}$ deben ser vistos al interior de un mismo marco estructural, tanto conceptual como terminológicamente.

El espacio de la teoría actor-red como propuesta teórica se sustenta en el interés de los teóricos organizacionales de explicar la distribución y ejercicio del poder entre organizaciones y entidades al interior de esferas particulares o redes. En este sentido, este enfoque argumenta que centrarse en preguntas sobre identidad, particularmente sobre auto-identidad, depende de la aceptación y reproducción de un set moderno de pre-

21 La irreversibilidad es un concepto de importancia en la estabilidad de los movimientos de la red (estos movimientos son los que Law (1997) denomina traducciones). La irreversibilidad establece la posibilidad de que las traducciones puedan cambiar a través del tiempo. Se logra la irreversibilidad inscribiendo intereses en artefactos tecnológicos y enrolando un gran número de actores en una red.

22 Los micro-actores son los individuos y los macro-actores son las instituciones, corporaciones, organizaciones gubernamentales, etc. (Callon, 1993). 
suposiciones y propone que la noción de agencia ofrece una perspectiva alternativa no moderna a partir de la cual explorar cómo las entidades o actores influyen en otros actores a través del proceso de traducción. ${ }^{23}$

Partiendo de la idea de que el conocimiento es interpretado, contingente y discutible (Fountain, 1999), esta teoría resalta dos temas importantes; por una parte, que el actor y la red no son sólo cosas a ver o aprehender por el investigador, sino que en sí mismos son marcos conceptuales, una forma de entender procesos sociales y técnicos; y por otra parte, que pensar en redes requiere concebir relaciones entre cosas en formas particulares.

La postura de la teoría actor-red podría resumirse como la resistencia a la deificación moderna de las limitaciones que evitan que el individuo vea las formas en las que lo "social" lo "técnico" y lo "natural" se interrelacionan en una suerte de red no tejida. ${ }^{24} \mathrm{~A}$ esto se agrega lo planteado por Michael (1996) en relación a que hay tres premisas teóricas a las cuales hay que adherirse a fin de producir una explicación del actor-red:

1 ro. Un agnosticismo generalizado, que requiere una imparcialidad analítica sobre los actores involucrados;

2do. Una simetría generalizada, que involucra el uso de un vocabulario abstracto y neutral para entender los puntos de vista conflictivos de los actores; y

23 La traducción representa los movimientos de la red (Law, 1997)

24 El concepto de "red no tejida" se refiere a la idea de que se mezclan ingredientes que forman una entidad homogénea y puede observarse el impacto de uno en particular, pero resulta absurdo ignorar arbitrariamente el efecto o interconexión de todos los ingredientes. Un ejemplo de esto es un bizcocho; los ingredientes se mezclan y puede que se "observe" el impacto del azúcar en la dimensión "dulzura", pero resulta determinante considerar los ingredientes como un todo para referirse al "bizcocho" propiamente. . 
3ro. Una asociación libre, que demanda el repudio de distinciones a priori entre lo social, lo natural y lo tecnológico.

La perspectiva del actor-red enfatiza la contingencia de las redes $^{25}$ y lo referido a sus cualidades emergentes; esto es, las redes no son de estabilidad prolongada e incluyen constantemente nuevos elementos que cambian las relaciones entre los actores. Por otra parte, la idea de las cualidades emergentes se refiere a evitar el requerimiento de imponer un marco teórico en la red.

Subyace la idea de exponer el trabajo realizado a fin de generar asociaciones y traducir más que apelar a constructos analíticos generales que realizarán todas las explicaciones y que estén basados en supuestos asumidos sobre el episteme moderno y que pueden ser "develados" en una redescripción de la teoría actor-red.

Es indiscutible el profundo impacto ontológico y epistemológico que implica este cambio propuesto por la teoría actorred. Por una parte, el cuestionamiento central viola el pilar ontológico fundamental de que la realidad depende de las subjetividades colectivas de una comunidad humana y, por otra, cuestiona el axioma epistemológico de que lo que llamamos conocimiento es construido por una comunidad humana. ${ }^{26}$

Esta teoría es un ensamblaje de historias modestas cuyas narrativas han cambiado de grandes historias con un orden cronológico a muchas historias pequeñas que forman un patrón sin posible cronología (Calas \& Smircich, 1999); lo que, ciertamente, la excluye del canon teórico modernista. Como

25 Esto es, que no son determinadas, permanentes o universales (Somerville, 1999)

26 Ciertamente, son estas presuposiciones, centrales a la sociología occidental y casi a todo el pensamiento post-kantiano, las que son cuestionadas por la teoría actor-red. 
tendencia teórica innova a partir de su reflexividad metodológica pues, no sólo se constituye en su objeto de interés, sino que también lo describe.

\section{Enfoques narrativos del conocimiento}

Al hablar de lo narrativo, el término ha sido usado en sentido amplio para referir el recuento de uno o más eventos ficticios (Denning, 2000; Prince, 1990). El significado de lo narrativo se crea estableciendo que algo es parte de un todo y que, usualmente, ese algo es la causa de algo más.

La idea que subyace a los enfoques narrativos es la renuncia a los modos ortodoxos de producción de conocimiento y la consideración de lugares alternativos a partir de los cuales se puede reconocer, investigar, analizar e interpretar el conocimiento. Esto, según Bacchilega (1997), responde a la noción tradicional que plantea que las perspectivas narrativas y la crítica intelectualmente rigurosa son yuxtaposiciones en contraste.

La narratividad es muchas veces asociada exclusivamente a la subjetividad y con ello se cuestiona su legitimidad en el marco del estricto rigor científico. Sin embargo, es a partir de la narración donde pueden reconocerse formas de producción que explican contextos (sociales, culturales, políticos y económicos) significativos operantes, aún cuando no hayan tenido cabida en la teorización tradicional. Esto se relaciona con las formas de manipulación y construcción narrativa, las cuales contribuyen a la posibilidad de diversos efectos ideológicos al interior de contextos sociales e históricos específicos.

La idea del performance escrito se abre espacio en los enfoques narrativos, lo que puede implicar reportar desde un ex- 
perimento o un cuestionario, hasta el relato de una historia; o realizar análisis narrativos de textos escritos y orales (documentos, emails, llamadas telefónicas, etc.). En los entornos organizacionales esto es importante porque muchos escritos de este tipo dan cuenta de problemáticas silenciadas o encubiertas, no necesariamente en forma intencional, y cuyo recuento sólo es posible desde el acto narrativo, porque la teoría formal tradicional rechaza la forma anecdótica con la que siempre se han vinculado. ${ }^{27}$

Tradicionalmente, la teorización organizacional ha sido criticada por su postura pretendidamente atemporal. Al abordar las problemáticas al interior de los entornos organizacionales, ha cultivado una postura inmanentista a la cual ha sido difícil renunciar. Esto se evidencia en las dificultades que históricamente ha enfrentado la teorización que aborda la multidisciplinariedad desde las ciencias sociales o que ha explícitamente generado espacios de reflexión de lo organizacional desde la experiencia social.

Se han identificado y desarrollado múltiples enfoques narrativos los que, de algún modo, incluyen no sólo las perspectivas de teorización abordadas previamente en este escrito (feminismo post-estructuralista, actor-red y análisis post-coloniales), sino que también amplían y subdividen otros grupos temáticos. Calas \& Smircich (1999) refieren la existencia de escritos experimentales que desvanecen las fronteras entre teoría y método, así como otros que presentan ilusiones de multivocidad. ${ }^{28}$ Otra agrupación de los enfoques narrativos existentes lleva a reconocer, al menos, siete aproximaciones diversas:

27 Es importante considerar que la narración tiene una directa asociación con la fabulación y que, en sí misma, existe tanto por lo que no es como por lo que es. Esto, indudablemente, quiebra en tanto postula lo que la teorización tradicional siempre ha rechazado.

28 Las autoras plantean que algunos de estos trabajos se centran en las convenciones de "escribir teoria" en el contexto de los estudios organizacionales. 
(1) Enfoques estructuralistas y lingüísticos. La narratología estructuralista pretende explicar la figuración narrativa en función de una gramática textual percibida. Esta posición asume que lo narrativo expresa una lógica genérica (poética) o un código genérico. Sin embargo, a fin de preservar este planteamiento se dio un giro importante, cambiando la estructura a favor de una descripción, lo que pretende abandonar la relación entre figuración y poética. Es así como surge este enfoque, a partir de la idea de que un concepto puede no servir propósitos descriptivos o analíticos y que, por tanto, puede ser generalmente aceptable reemplazarlo con otro concepto que esté mejor formulado.

En los teóricos de esta tendencia (Bal, 1997; Toker, 1993; Genette, 1988, 1997) resalta el planteamiento de la importancia del concepto de la estructura de las ideas, de una matriz teórica o un discurso. Sin embargo, se reconoce que la búsqueda de un set definitivo de conceptos es ilimitada, por lo que se hace necesaria la constante re-descripción, reciclando viejos conceptos a través del uso de nuevos lenguajes que reemplacen el vocabulario desgastado.

Subyace como central la interrelación entre el cómo y porqué de la experiencia, y el cómo y porqué de la forma en que se articula su descripción, porque el significado atribuido a la experiencia puede variar y, consecuentemente, debiera variar la forma en que lingüísticamente se articula su descripción.

(2) Recuentos retóricos, bakhtinianos y fenomenológicos. En el marco de la subjetividad, estos recuentos abordan el intercambio de enunciados como una forma dialógica, donde el análisis de textos siempre es intertextual porque las formas de construcción del todo siempre son interdependientes de muchos textos. De ahí surge la porosidad y la flexibilidad entre los géneros de construcción a fin de que pueda lograrse un 
diálogo entre géneros literarios que permita construcciones de mayor complejidad (Aczel, 1998; Boardman, 1992; Pelan, 1989, 1996).

Resaltan los argumentos fenomenológicos de Bakhtin, los denominados postulados sobre filosofía de la cultura. ${ }^{29}$ En éstos aborda la condición de la lucha de la vida histórica de la cultura, la persona y la humanidad, centrándose en las relaciones sujeto/objeto, sujeto/otro sujeto, los problemas de la intersubjetividad, la conciencia subjetiva y la subjetividad ontológica. Estos postulados son importantes para la teorización narrativa ya que persiguen encontrar alternativas a los enfoques estructuralistas que ven la teorización como un todo hermético, auto-suficiente, en un sistema cerrado sin nada más allá de sí mismo.

(3) Enfoques interdisciplinarios. Estos enfoques persiguen discutir los fenómenos operantes y las implicaciones que éstos revisten en la comprensión de la representación, la semántica, el lenguaje, la ciencia cognitiva y la vida artificial, la teoría de los mundos posibles, y el estudio de los hipertextos.

En materia de teoría de los mundos posibles, resalta el trabajo de Dolezel $(1990,1995)$, que explora la posibilidad de aplicación de la teoría de los mundos posibles a la semántica literaria. Al revisar el trabajo de Dolezel, Ryan (1998) sostiene que los mundos ficcionales son mundos posibles construidos por el lenguaje a través de una fuerza performativa y que estos mundos difieren ontológicamente del mundo real en su na-

29 Su proyecto filosófico deriva fundamentalmente de la tradición de la estética de la Ilustración. Para Bakhtin, lo importante era la actividad estética como modo de producción de relaciones intersubjetivas recíprocas, necesarias para producir unidad intima de individuos cuya especificidad no se ve amenazada. Lo estético es el marco en el cual se manifiesta la esencia del logro del héroe, separada del acto abierto de ser y concretada por la virtud de la exterioridad del autor. 
turaleza incompleta, aún cuando sean realidades autónomas creadas mediante el poder del lenguaje de la ficción.

Respecto de la ciencia cognitiva, se abordan tópicos cuya emergencia responde a la necesidad actual de interdisciplinariedad que enfrentan las ciencias como resultado de la ruptura teórica que representa la postmodernidad. Entre estos tópicos se encuentran los enfoques de la cognición basados en sistemas teóricos y neurociencia computacional, los enfoques cognitivos evolutivos y basados en inteligencia artificial (Hayles, 1999; Cook, 1994; Ryan, 1991), las implicaciones (epistemológicas) de la percepción y la representación, el problema de la representación del conocimiento, la autopoiesis, las implicaciones para la epistemología y la filosofía de la ciencia (Manfred, 1997; Spolsky, 1993; Bizzell, 1982).

Finalmente, una de las tendencias de mayor controversia es el estudio de los hipertextos (Hayles, 1997; December, 1996; Thomas, 1993), ya que su emergencia viene de la mano de la creación de la cultura del cyborg, y sus implicaciones respecto de las formas tradicionales de escritura. Esto se relaciona con el posicionamiento de la narración y el narrador, ya que el lugar del enunciador se vuelve difuso y responde a espacialidades y temporalidades ambiguas que traicionan la distinción entre el autor y su obra.

(4) Narratología postmodernista. A partir de las preguntas que se plantea la teorización postmodernista, la actual evolución y desarrollo de la teorización que aborda lo narrativo se ha visto cuestionada por no poder enmarcarse dentro de una única historia continua. En este sentido, los teóricos (Richardson, 1997, 2000a; Heise, 1997; Brooke-Rose, 1991) tratan de dar sentido y espacio a figuras olvidadas, cabos sueltos e hilos conductores inconexos.

Esta corriente de teorización se centra en la retención del rigor analítico de los enfoques tradicionales, pero igualmen- 
te se desplaza lejos de los parámetros relativamente limitados del estructuralismo y aborda nuevas preguntas que surgen de los textos y posturas postmodernistas. Esto, según Richardson $(2000 \mathrm{~b})^{30}$, ha llevado a re-pensar radicalmente los conceptos fundacionales de la teoría narrativa.

(5) Enfoques ideológicos. Estos enfoques quiebran la teorización tradicional a partir de la generación de una forma de teorización alternativa que incluye, principalmente, el cruce de variables que descubren estadios en las formas y espacios en que se articulan los discursos del conocimiento y las segregaciones que éstos generan a partir de los sujetos que los articulan y a quienes estos discursos representan y excluyen. Entre estos enfoques se incluyen los escritos sobre teoría feminista y de género (Itzin, 1995; Homans, 1994; Winnett, 1990; Hite, 1989), teoría gay, lesbiana y queer (Roof, 1996; Farwell, 1996; Bersani, 1995; Lanser, 1995;), etnicidad y raza (Frable, 1997; Warhol, 1995; Stepto, 1991), los enfoques históricos marxistas y el nuevo historicismo (Ginsburg, 1996; Quint, 1993), y los análisis post-coloniales (Ashcroft, Griffiths \& Tiffin, 1998; Spurr, 1993).

(6) Enfoques psicológicos. Un espacio significativo se les ha otorgado a los enfoques que utilizan la psicología como base conceptual para abordar la construcción del conocimiento. Esto, principalmente, por la naturaleza anárquica con la que ha sido asociado el movimiento conceptual postmodernista. En este sentido, uno de los grandes aportes de la psicología a la teorización postmodernista se enmarca en la re-conceptualización de la "desviación"; que incluye las formas en

30 Para Richardson, la narrativa es una representación de una serie de eventos causalmente relacionados, entendiendo "causalmente relacionados" como "normalmente conectados". Esto implicaría que incluye las narrativas verbales y no verbales. Esto supone que muchos elementos no-narrativos residen al interior de un marco estructural narrativo mayor. Podría decirse que esto rompe con la idea del carácter exclusivamente narrativo de lo narrativo. 
que se teoriza respecto de lo normal y lo anormal (Ferrell \& Websdale, 1999; Bronfen, 1998), especialmente a partir de un cuestionamiento de las explicaciones biológicas y fisiológicas de la desviación, así como su construcción psicológica ${ }^{31}$. Por otra parte, esta teorización ha sido una de las bases principales para los debates respecto de los mecanismos de interacción, socialización y control social (Adler \& Adler, 2002; McWhorter, 1999; Trew \& Kremer, 1998; Nicolson, 1996), que tanto preocupan a los teóricos postmodernistas.

Esto es de especial importancia porque la forma en que se entienden la invisibilización, la exclusión, la subordinación, entre otros, ha pasado a convertirse en algo que no se centra exclusivamente en el debate sobre el poder y su relación con la propiedad material, sino que también considera las formas de poder que son definidas desde lo conceptual.

(7) Enfoques post-estructuralistas. Estos enfoques se centran en el uso de la tropología en tanto teoría y práctica de interpretación del significado figurativo (Mellard, 1987). Como ya fue sugerido al hablar de teorización feminista post-estructuralista, el post-estructuralismo utiliza la invención y desarrollo de críticas de la metafísica de la presencia y el logocentrismo que fundamenta el proyecto filosófico-científico tradicional, basándose principalmente en el lenguaje como sistema material-semiótico de diferencias que simultáneamente está sujeto a fuertes limitaciones y posibilidades cuasi-infinitas de afirmación, experimentación e invención. En este sentido, la idea de los enfoques post-estructuralistas es el quiebre de lo que reconoce como un historicismo radical para dar espacio a formas más flexibles que reconocen que el juicio y la verdad no pueden ser universales (Grumley, 1989).

31 En este aspecto, es de especial relevancia el papel que ha tenido la perspectiva psicoanalítica en la explicación de la desviación. 


\section{Reflexiones Iniciales}

La teorización organizacional postmodernista representa esfuerzos por trascender las limitaciones metodológicas del positivismo, ya que centra su preocupación en comprender las formas en que los sujetos adscriben significados a sus actividades diarias y a la vida organizacional en sí misma, sin invalidar el locus enunciativo ni la forma de enunciación utilizada.

Sin embargo, el hecho de que uno de los problemas atribuidos a la condición postmodernista es la ambigüedad de su terminología, ha provocado que en la administración y en los estudios organizacionales no haya tenido tanto protagonismo (Cooper \& Burrell, 1988) y recién se esté debatiendo en los espacios legitimados de teorización. Lo anterior podría ser el resultado del juego postmodernista de los múltiples significados. 32

Prior-Miller (1989) ha sugerido que hay cuatro perspectivas científicas sociales importantes que constituyen el marco estructural para la mayoría de la teoría e investigación organizacional contemporánea: el interaccionismo simbólico, las teorías de intercambio, las teorías de conflicto y las teorías estructurales-funcionales. Sin embargo, estos cuatro enfoques comparten presuposiciones fundacionales similares, como el hecho de no identificar sujetos y entidades organizacionales individuales con distintos límites, lo que se relaciona con el

32 Y la teorización siempre ha buscado "el" significado que permita categorizar los fenómenos que operan al interior de los entornos organizacionales. Una primera consideración es que esos juegos postmodernistas operan como una forma de resistencia a esa misma teorización homogeneizante. Por otra parte, no puede obviarse que la teorización modernista tiene como tendencia la articulación de reglas generales sobre la sociedad, el conocimiento y la verdad. El modernismo sostiene la primacía de la razón por sobre la irracionalidad, apelando a leyes universales o principios generales, los que suponen que la ciencia y el conocimiento son acumulativos y, por ende, progresivos. 
episteme moderno, que homogeneiza la teoría a partir de una homogeneización de la teorización.

A partir de lo anterior, se pueden reconocer, al menos, tres problemáticas:

1ro. Una preferencia por el pensamiento, en detrimento de otras formas de conocimiento.

2do. Una distorsión que favorece la lógica binaria.

3ro. La desestimación del sentimiento (emociones y moral) en la comprensión, razonamiento y toma de decisiones.

Hancock \& Tyler (2001) plantean que, la naturaleza de la realidad organizacional ha sido comprendida, administrada y dirigida sobre la base de proposiciones filosóficas positivistas, combinadas con los imperativos sistémicos del análisis funcionalista. Esto ha significado una visión restringida, ya que no sólo no se consideran los aspectos informales del comportamiento organizacional, sino que éstos son tratados como aspectos irracionales que deben ser erradicados mediante la imposición de mecanismos regulatorios formulados racionalmente.

Los estudios organizacionales postmodernistas ilustran las consecuencias para las ciencias sociales del cuestionamiento de valores y el relativismo, llevando a una crítica más que a una construcción (Rosenau, 1992). La idea central de la teorización postmodernista es que, si bien ésta se constituye en función del modernismo, no es su intención que la ciencia busque conocimiento o verdad, sino que explique los fenómenos operantes en el marco de los contextos culturales. ${ }^{33}$

33 Los estudios y teorización organizacionales han históricamente respondido a nociones que conciben las organizaciones como entidades objetivas, lejanas de los fenómenos naturales que existen en el "mundo real". 
Resulta fundamental comprender que aun cuando esta teorización postula la muerte de la razón, no asume el nacimiento de la sinrazón, sino más bien el reconocimiento de las ambigüedades y las incertidumbres que permiten dar cuenta de que el conocimiento no puede ni debe ser universalizado y que, por el contrario, resulta más enriquecedor celebrar las diferencias.

El argumento de Gergen (1992), que sostiene que los modelos tradicionales de organización deben ser entendidos como el resultado de una combinación de dos cuerpos hegemónicos de discurso (romanticismo y modernismo), es coherente con el quiebre planteado por el postmodernismo que, según este autor, se centra en tres proposiciones interrelacionadas sobre la naturaleza del conocimiento (el reemplazo de lo real por lo representacional, la comprensión de la representación como un "artefacto comunal” y la necesidad de una auto-reflexión irónica).

Cada uno de los enfoques analizados ofrece contribuciones específicas a la teorización organizacional en tanto, como paradigmas, se centran en acciones reflexivas que pretenden comprender la construcción de conocimiento en la teorización organizacional; esto es, no determinan si un paradigma es correcto o no, sino cuán bueno es en explicar o delimitar la experiencia a partir de la cual se construye la realidad organizacional. Por otra parte, es importante el potencial reconocido por esta teorización en las intertextualidades y las intersecciones con las que se realizan transformaciones re-interpretativas y re-creativas del conocimiento. ${ }^{34}$

Este estudio de la naturaleza de lo que consideramos conocimiento a partir de la textualidad resulta fundamental al ha-

34 Esto podria asociarse con la primera proposición de Gergen (1992), sobre el reemplazo de lo real por lo representacional. 
blar de la coherencia de la teorización. La tradición académica hegemónica ha negado un espacio significativo a subalternos y marginados; sin embargo, todos son parte del discurso globalista y operan en organizaciones que en ningún caso son ajenas al fenómeno.

Ahora bien, si bien esto podría sugerir que a partir del desarrollo de estos paradigmas es cuando recién puede iniciarse el debate sobre cómo afecta la temporalidad y espacialidad a las dinámicas organizacionales, un cuestionamiento más fundamental se centra en las formas en que la globalización, como fenómeno discursivo, aborda la integración y caracteriza la condición global. ${ }^{35}$

Esa "naturaleza de nuestro presente" que refiere Foucault (1984:34-37) se logra a partir del cuestionamiento que coloca los supuestos bajo escrutinio. Se reconoce en el postmodernismo la apertura de una forma audaz de enfrentar el conocimiento, un atreverse a saber que está permitiendo aproximarse a los límites de lo que se es y se construye organizacionalmente y a la posibilidad de ser y construirse en forma distinta. ${ }^{36}$

En este sentido, las tendencias descritas mantienen el foco en la heterogeneidad del pensamiento y plantean un importante punto de partida en la necesaria evolución de la teorización organizacional hacia espacios que permitan ampliar el espectro analítico para incluir experiencias silenciadas, obviadas e inadvertidas, y para centrarse en un enfoque que complementa lo descriptivo con lo crítico y se pregunta no sólo respecto de lo que se ve, sino a qué responde cen-

35 Esto podría relacionarse con la segunda proposición de Gergen (1992), sobre la comprensión como un "artefacto comunal".

36 Esto se relacionaría con la tercera proposición de Gergen (1992), referida a la necesidad de una auto-reflexión irónica. 
trarse en la búsqueda específica de ciertos fenómenos. ${ }^{37} \mathrm{Su}$ importancia también radica en el imperativo de recodificar la sustancia que otorga sentido a la experiencia de las personas en las organizaciones y cómo ésta convive en medio de la experiencia colectiva.

Paolini (2000) plantea que la postmodernidad es con frecuencia utilizada para describir condiciones de dislocación y flujo, "un desenmascaramiento de espacio y tiempo" (p. 42) que genera una suerte de incertidumbre y contingencia. Evidentemente, los códigos estructurales de la teorización organizacional postmodernista responden a una necesidad de desenmascarar el canon teórico establecido, lo que llevaría a un cuestionamiento con relación a qué corresponde hacer con las categorías y sistemas con que han operado históricamente y que han regulado y definido la construcción de teoría y acción organizacional.

Interesantemente, estas propuestas no han sido articuladas propiamente en el campo de los estudios organizacionales tradicionales, sino que surgen principalmente de las tendencias teóricas críticas de la postmodernidad en los campos de la literatura, los estudios culturales, y los estudios alternativos. Esto es importante para reconocer la inminente transición desde la multi e interdisciplinariedad hacia la transdisciplinariedad, que resulta tan necesaria a fin de enriquecer los análisis. ${ }^{38}$

Una reflexión final importante llamaría a desarrollar un espacio de debate significativo en materia de estudios crí-

37 Esto se relaciona con lo planteado por Althusser (1971) respecto de las formas en que los agentes son "interpelados" a fin de reconocer e identificarse con ciertas posiciones de los sujetos que los atan a ciertas definiciones morales y cientificas sobre quiénes son.

38 Esto es, ciertamente, un paso significativo en el quiebre del inmanentismo referido previamente. 
ticos de la administración en tanto eje fundamental de las dinámicas organizacionales. Los niveles de micro y macro análisis que históricamente se han desarrollado sirven a un propósito mayormente de funcionalidad empírica; sin embargo, no resuelven el problema de que las categorizaciones operantes resultan obsoletas frente al cambio paradigmático de la teorización filosófica sobre el origen y funcionalidad de las ideas. Lo importante no es el posicionamiento acomodado de explicaciones relativas a las dinámicas operantes en las organizaciones, sino el estudio de la experiencia y funcionalidad a partir de posturas y posibles categorizaciones críticas no condescendientes.

\section{Referencias Bibliográficas}

Aczel, R. 1998. Hearing Voices in Narrative Texts. New Literary History. 29. 467-500.

Adler, P. \& Adler, P. 2002. Constructions of Deviance: Social Power, Context, and Interaction. Scarborough, ON (Canada). Nelson Thomson Learning.

Althusser, L. 1971. Lenin and Philosophy and Other Essays. New York. Monthly Review Press.

Alvesson, M. 1995. The meaning and meaningless of postmodernism: some ironic remarks. Organization Studies. 15 (6). 1047-1075.

Alvesson, M. \& H. 1996. Making Sense of Management. Londres. Sage.

Ashcroft, B; G. Griffiths \& H. Tiffin. 1998. Key Concepts in Post-Colonial Studies. Londres. Routledge.

Bacchilega, C. 1997. Postmodern Fairy Tales: Gender and Narrative Strategies. Philadelphia. University of Pennsylvania Press.

Bal, M. 1997. Narratology: Introduction to the Theory of Narrative. Toronto. University of Toronto Press.

Bersani, L. 1995. Homos. Cambridge, MA. Harvard University Press. 
Bizzell, P. 1982. Cognition, Convention and Certainty: What we Need to Know About Writing. Pre-Text. 3. 213-244.

Boardman, M. M. 1992. Narrative Innovation and Incoherence. Durham, NC. Duke University Press.

Bronfen, E. 1998. The Knotted Subject: Hysteria and its Discontents. Princeton, NJ. Princeton University Press.

Brooke-Rose, C. 1991. Stories, Theories, and Things. Cambridge, MA. Cambridge University Press.

Burrell, G. 1994. Modernism, postmodernism and organizational analysis: The contributions of Jürgen Habermas. Organization Studies. 15 (1). 1-45.

Burrell, G. 1996. Paradigms, metaphors, discourses, genealogies. En S. Clegg, C. Hardy \& W. Nord (eds.) Handbook of Organization Studies. Londres. Sage.

Burrell, G. 1997. Pandemonium: Towards a retro-organization theory. Londres. Sage.

Calas, M. \& L. Smircich (1999): Past modernism? Reflections and tentative directions, Academy of Management Review. 24 (4). 649671.

Callon, M. 1986. Some Elements of a Sociology of Translation: Domestication of the Scallops and the Fishermen. En J. Law (ed.) Power, Action and Belief: A New Sociology of Knowledge. Londres. Routledge \& Kegan Paul.

Callon, M. 1993. Variety and irreversibility in networks of technique conception and adoption. En D. Foray \& C. Freeman (eds.) Technology and the Wealth of Nations: The dynamic of constructed advantage. Londres. Pinter.

Callon, M. \& B. Latour. 1981. Unscrewing the big Leviathan. En K. D. Knorr-Cetina \& M. Mulkay (eds.) Advances in Social Theory and Methodology. Londres. Routledge and Kegan Paul.

Castro-Gómez, S. 1998. Manifiesto Inaugural del Grupo Latinoamericano de Estudios Subalternos. En S. Castro-Gómez \& E. Mendieta. Teorías sin disciplina (Latinoamericanismo, poscolonialidad y globalización en debate). México. Miguel Angel Porrúa. 
Chafetz, J. S. 1988. Feminist Sociology: An Overview of Contemporary Theories. Ithaca, IL. F. E. Peacock.

Chia, R. 1997. From modern to postmodern organizational analysis. Organization Studies. 16 (4). 579-604.

Clegg, S. R. 1993. Modern Organizations: Organization Studies in a Postmodern World. Londres. Sage.

Cook, G. 1994. Discourse and Literature: The Interplay of Form and Mind. Oxford. Oxford University Press.

Cooper, R. \& G. Burrell. 1988. Modernism, Postmodernism and Organizational Analysis: An Introduction. Organization Studies. 9 (1). 91-112.

Crook, S.; J. Pakulski \& M. Waters. 1992. Postmodernization: Change in Advanced Societies. Londres. Sage.

Darby, P. (ed.) 2000. At the Edge of International Relations. Postcolonialism, Gender, and Dependency. Londres. Continuum.

December, J. 1996. Living in Hypertext. EJournal. 6 (3).

Deleuze, G. \& F. Guattari. 1988. A thousand plateaus: Capitalism and schizophrenia. Londres. Althone.

Dellamora, E. (ed.) 1995. Postmodern Apocalypse. Theory and Cultural Practice at the End. Philadelphia. University of Pennsylvania Press.

Denning, S. 2000. The Springboard: How Storytelling Ignites Action in Knowledge-Era Organizations. Boston, MA. Butterworth Heinemann.

Dolezel, L. 1990. Occidental Poetics: Tradition and Progress. Lincoln, NE. University of Nebraska Press.

Dolezel, L. 1995. Fictional Worlds: Density, Gaps and Inference. Style. 29. 201-214.

Donovan, J. 1996. Feminist Theory. New York. Continuum.

Duran, J. 1998. Philosophies of Science/Feminist Theories. Boulder, CO. Westview Press.

Farwell, M. 1996. Heterosexual Plots and Lesbian Narrative. New York. New York University Press. 
Ferrell, J. \& Websdale, N. (eds.) 1999. Making Trouble: Cultural Constructions of Crime, Deviance, and Control. New York. De Gruyter.

Fondas, N. 1997. Feminization unveiled: Management qualities in contemporary writings. Academy of Management Review. 22. 257-283.

Foucault, M. 1984. What is Enlightment? En P. Rabinow (ed.) The Foucault Reader. New York. Pantheon.

Fountain, R. 1999. Socio-scientific issues via actor theory network. Journal of Curriculum Studies. 31 (3). Mayo / Junio. 339-358.

Fox, S. 2000. Communities of practice, Foucault and actor-network theory. The Journal of Management Studies. 37 (6). Septiembre. 853-867.

Frable, D. E. S. 1997. Gender, racial, ethnic, sexual and class identities. Annual Review of Psychology. 48. 139-142.

Friedman, J. 2001. Identidad cultural y proceso global. Buenos Aires. Amorrortu.

Genette, G. 1988. Narrative Discourse Revisited. Ithaca, NY. Cornell University Press.

Genette, G. 1997. Paratexts: On the Threshold of Interpretation. Cambridge, MA. Cambridge University Press.

Gergen, K. 1992. Organization Theory in the Postmodern Era. En M. Reed \& M. Hughes (eds.) Rethinking Organization: New Directio$n s$ in Organization Theory and Analysis. Londres. Sage.

Gibson, A. 1996. Towards a Postmodern Theory of Narrative. Edimburgo. Edinburgh University Press.

Ginsburg, M. P. 1996. Economics of Change: Form and Transformation in the Nineteenth-Century Novel. Stanford. Stanford University Press.

Glucksmann, A. 1997. La estupidez. Ideologías del postmodernismo. Barcelona. Ediciones Península.

Grumley, J. 1989. History and Totality: Radical Historicism from Hegel to Foucault. Londres. Routledge. 
Habermas, J. 1975. Legitimation Crisis. Boston, Mass. Beacon.

Hancock, P. \& M. Tyler. 2001. Work, Postmodernism and Organization. Londres. Sage.

Harding, S. 1990. Feminism, Science, and the Anti-Enlightenment Critique. En L. J. Nicholson (ed.) Feminism/Postmodernism. New York. Routledge.

Harding, S. 1993. The Science Question in Feminism. Ithaca, NY. Cornell University Press.

Hayles, N. K. (ed.) 1997. Technocriticism and Hypernarrative. Special Issue. Modern Fiction Studies. 43 (3).

Hayles, N. K. 1999. How We Became Posthuman: Bodies in Cybernetics, Literature, and Informatics. Chicago. University of Chicago Press.

Hawthorn, J. 1992. A Glossary of Contemporary Literary Theory. Londres. Edward Arnold.

Heise, U. K. 1997. Chronoschisms: Time, Narrative, and Postmodernism. Cambridge, MA. Cambridge University Press.

Heydebrand, W. V. 1989. Work and the Nature of Man. Londres. Crosby Lockwood Staples.

Hite, M. 1989. The Other Side of the Story: Structures and Strategies of Contemporary Feminist Narratives. Ithaca, NY. Cornell University Press.

Homans, M. 1994. Feminist Fictions and Feminist Theories of Narrative. Narrative. 2. 3-16.

Hopenhayn, M. 1992. ¿Pensar lo social sin planificación ni revolución? Revista de la CEPAL. No. 48.

Hopenhayn, M. 2000. Identidad diseminada-identidad desintegrada: opciones abiertas. En http://www.mav.cl/foro_cult/hopenhayn01. htm

Itzin, C. 1995. The gender culture in organizations. En C. Itzin \& J. Newman (eds.) Gender, culture and organizational change: putting theory into practice. Londres. Routledge.

Jameson, F. 1991. Ensayos sobre el Posmodernismo. Buenos Aires. Imago Mundi. 
Kaghan. W. N. \& G. C. Bowker. 2001. Out of machine age?: Complexity, sociotechnical systems and actor network theory. Journal of Engineering and Technology Management. 18 (3,4). Septiembre-Diciembre. 253-269.

Krausz, M. (ed.) 2002. Is There a Single Right Interpretation? University Park, Pennsylvania. The Pennsylvania State University Press.

Lanser, S. S. 1995. Sexing the Narrative: Propriety, Desire, and the Engendering of Narratology. Narrative. 3. 85-94.

Lash, S. 1988. Postmodernism as a Regime of Signification. Theory, Culture \& Society. 5 (2-3). 311-336.

Latour, B. 1992. Where are the Missing Masses? The Sociology of Some Mundane Artifacts. En W. E. Bijeker \& J. Law (eds.) Shaping Technology / Building Society. Cambridge, MA. MIT Press.

Law, J. 1992. Notes on the theory of the actor-network: ordering, strategy and heterogeneity. Systems Practice. 5. 379-393.

Law, J. 1997. Traduction/trahison: Notes on ANT. Departamento de Sociología y Antropología Social. Keele University. Keele, Reino Unido.

Law, J. \& J. Hassard (Eds.) 1999. Actor Network Theory and After. Malden, MA. Blackwell.

López Gil, M. 1993. Filosofía, Modernidad y Posmodernidad. Buenos Aires. Editorial Biblos.

Lyon, D. 1997. Postmodernidad. Madrid. Alianza Editorial.

Lyotard, J. 1984. The Postmodern Condition: A Report on Knowledge. Minneapolis. University of Minnesota Press.

Manfred, J. 1997. Frames, Preferences, and the Reading of Third-Person Narrative: Towards a Cognitive Narratology. Poetics Today. 18. 441-468.

Mellard, J. M. 1987. Doing Tropology: Analysis of Narrative Discourse. Urbana, IL. University of Illinois Press.

Michael, M. 1996. Constructing Identities. Londres. Sage.

McWhorter, L. 1999. Bodies and Pleasures: Foucault and the Politics of Sexual Normalization. Indiana. Indiana University Press. 
Nicolson, P. 1996. Gender, power and organisation: a psychological perspective. Londres. Routledge.

Ong, A. 1996. Anthropology, China and Modernities: the Geopolitics of Cultural Knowledge. En H. L. Moore (comp.) The Future of Anthropological Knowledge. Londres. Routledge.

Paolini, A. 2000. Globalization. En P. Darby (ed.) At the Edge of International Relations. Postcolonialism, Gender and Dependency. New York. Continuum.

Parker, M. 2000. Organizational Culture and Identity. Londres. Sage.

Phelan, J. 1989. Reading People, Reading Plots. Character, Progression, and the Interpretation of Narrative. Chicago. University of Chicago Press.

Phelan, J. 1996. Narrative as Rhetoric: Technique Audiences, Ethics, Ideology. Columbus, $\mathrm{OH}$. Ohio State University Press.

Prince, G. 1990. A Dictionary of Narratology. Lincoln, NE. University of Nebraska Press.

Prior-Miller, M. 1989. Four major social scientific theories and their value to the public relations researcher. En C. Botan \& V. Hazleton. Public Relations Theory. Hillsdale, NJ. Lawrence Erlbaum.

Putnam, L. 1996. Situating the author and text. Journal of Management Inquiry. 5. 382-386.

Quint, D. 1993. Epic and Empire: Politics and Generic Form from Virgil to Milton. Princeton, NJ. Princeton University Press.

Richardson, B. 1997. The Other Reader's Response: On Multiple, Divided, and Oppositional Audiences. Criticism. 39. 31-53.

Richardson, B. 2000a. Narrative Poetics and Postmodern Transgression: Theorizing the Collapse of Time, Voice, and Frame. Narrative 8. 23-42.

Richardson, B. 2000b. Recent Concepts of Narrative and the Narratives of Narrative Theory. Style. Verano. En http://www.findarticles. com/cf_0/m2342/2_34/68279069/print.jhtml

Roy, P. 2001. At home in the world? The gendered cartographies of globality. Feminist Studies. 27 (3). 709-731. 
Roof, J. 1996. Come as You Are: Sexuality and Narrative. New York. Columbia University Press.

Rosenau, P. M. 1992. Post-Modernism and the Social Sciences. Insights, Inroads, and Intrusions. Princeton, NJ. Princeton University Press.

Ryan, M. 1991. Possible Worlds, Artificial Intelligence, and Narrative Theory. Bloomington, IN. Indiana University Press.

Ryan, M. 1998. Heterocosmica: Fiction and Possible Worlds (Review). Style. Otoño. En http://www.findarticles.com/cf_0/m2342/3_ 32/55082390/print.jhtml

Scott, D. 1999. Refashioning Futures: Criticism after Postcoloniality. Princeton, NJ. Princeton University Press.

Smith, B. G. 1995. Gender, Objectivity and the Rise of Scientific History. En W. Natter; T. R. Schatzki \& J. P. Jones III. Objectivity and Its Other. New York. The Guilford Press.

Somerville, I. 1999. Agency versus identity: actor-network meets public relations. Corporate Communications. 4 (1). 6-13.

Spender, J. 1998. Pluralist epistemology and the knowledge-based theory of the firm. Organization. 5. 233-256.

Spivak, G. C. 1988. Can the Subaltern Speak? En C. Nelson \& L. Grossberg (eds.) Marxism and the Interpretation of Culture. Urbana, IL. University of Illinois Press.

Spolsky, E. 1993. Gaps in Nature: Literary Interpretation and the Modular Mind. Albany, NY. State University of New York Press.

Spurr, D. 1993. The Rhetoric of Empire. Colonial Discourse in Journalism, Travel Writing, and Imperial Administration. Durham, NC. Duke University Press.

Stark, D. 2001. Actor Network Theory and After. Contemporary Sociology. 30 (1). Enero. 96-97

Stepto, R. B. 1991. From Behind the Veil: A Study of Afro-American Narrative. Urbana, IL. University of Illinois Press.

Hager, T. 1993. A Rhetoric of Hypertext Inventio. Writing on the Edge. 4. 103-115. 
Taylor, M. C. 1984. Erring: a Postmodern A/theology. Chicago. University of Chicago Press.

Thompson, P. 1993. Fatal distraction. En J. Hassard \& M. Parker (eds.) Postmodernism and Organizations. Londres. Sage.

Toker, L. 1993. Eloquent Reticence: Withholding Information in Fictional Narrative. Lexington, KY. University Press of Kentucky.

Touraine, A. 1990. Modernity and the Subject. Paper presentado en el Congreso de la Asociación Sociológica Internacional. Julio 10.

Trew, K. \& J. Kremer. 1998. Gender \& Psychology. Londres. Arnold.

Van Maanen, J. 1988. Tales of the Field. Chicago. University of Chicago Press.

Warhol, R. R. 1995. 'Reader, Can You Imagine? No, You Cannot': The Narratee as Other in Harriet Jacob's Text. Narrative. 3. 57-72.

Weedon, C. 1987. Feminist Practice and Poststructuralist Theory. Oxford. Basil Blackwell.

Winnett, S. 1990. Coming Unstrung: Women, Men, Narrative, and Principles of Pleasure. PMLA. 105. 505-518. 